\title{
Structural mechanism for the arginine sensing and regulation of CASTOR1 in the mTORC1 signaling pathway
}

\author{
Zhongchao Gai ${ }^{1}$, Qian Wang ${ }^{2}$, Can Yang ${ }^{1}$, Lei Wang ${ }^{1}$, Wei Deng ${ }^{2}$, Geng Wu ${ }^{1}$ \\ ${ }^{I}$ State Key Laboratory of Microbial Metabolism, School of Life Sciences \& Biotechnology, Shanghai Jiao Tong University, \\ Shanghai, China; ${ }^{2}$ National Center for Protein Science Shanghai, Shanghai, China
}

The mTOR complex I (mTORC1) signaling pathway controls many metabolic processes and is regulated by amino acid signals, especially arginine. CASTOR1 has been identified as the cytosolic arginine sensor for the mTORC1 pathway, but the molecular mechanism of how it senses arginine is elusive. Here, by determining the crystal structure of human CASTOR1 in complex with arginine, we found that an exquisitely tailored pocket, carved between the NTD and the CTD domains of CASTOR1, is employed to recognize arginine. Mutation of critical residues in this pocket abolished or diminished arginine binding. By comparison with structurally similar aspartate kinases, a surface patch of CASTOR1-NTD on the opposite side of the arginine-binding site was identified to mediate direct physical interaction with its downstream effector GATOR2, via GATOR2 subunit Mios. Mutation of this surface patch disrupted CASTOR1's recognition and inhibition of GATOR2, revealed by in vitro pull-down assay. Normal mode (NM) analysis revealed an 'open'-to-'closed' conformational change for CASTOR1, which is correlated to the switching between the exposing and concealing of its GATOR2-binding residues, and is most likely related to arginine binding. Interestingly, the GATOR2-binding sites on the two protomers of CASTOR1 dimer face the same direction, which prompted us to propose a model for how dimerization of CASTOR1 relieves the inhibition of GATOR1 by GATOR2. Our study thus provides a thorough analysis on how CASTOR1 recognizes arginine, and describes a possible mechanism of how arginine binding induces the inter-domain movement of CASTOR1 to affect its association with GATOR2.

Keywords: mTORC1 signaling pathway; arginine; CASTOR1; GATOR2; GATOR1; crystal structure

Cell Discovery (2016) 2, 16051; doi:10.1038/celldisc.2016.51; published online 27 December 2016

\section{Introduction}

The target of rapamycin (TOR) pathway (known as mammalian TOR, mTOR, pathway in mammals) is a central signal transduction pathway regulating many metabolic processes such as protein translation, lipid synthesis, autophagy, and so on in a vast variety of organisms from yeast to human. Aberrant regulation of the mTOR pathway in human predisposes to many diseases including cancer, diabetes, and autoimmune diseases $[1,2]$. The mTOR pathway is under stringent regulation by various upstream nutrient signals such as amino acids [3, 4]. Amino acid availability determines the nucleotide bound state of the heterodimeric Rag GTPases consisting of RagA or RagB in complex with

Correspondence: Geng Wu

Tel: +86-021-34205914; Fax: +86-021-34207025;

E-mail: geng.wu@sjtu.edu.cn.

Received 28 October 2016; accepted 12 December 2016
RagC or RagD [5]. Sufficient supply of amino acids promotes GTP-binding for RagA/RagB and GTP hydrolysis to GDP for $\mathrm{RagC} / \mathrm{RagD}$, which in turn recruits the mTOR complex I (mTORC1) (consisting of mTOR, Raptor, and mLST8) to its lysosomal anchor, the pentameric Ragulator complex [6, 7]. The mTORC1 complex is then activated by the lysosome-located small GTPase Rheb [8, 9], and phosphorylates a vast spectrum of downstream targets including ribosomal S6 kinase 1 (S6K1) and eIF-4E-binding protein 1 (4E-BP1).

As a key player for transmitting amino acid signals, the Rag proteins are regulated by multiple distinct factors, such as the Ragulator complex which serves as a guanine nucleotide exchange factor (GEF) for RagA/RagB [7], the FLCN/FNIP2 complex, which is a GTPase-activating protein (GAP) for $\operatorname{RagC/RagD}[10,11]$. The trimeric GATOR1 complex, comprising of DEPDC5, NPRL2, and NPRL3, functions as a GAP for RagA and RagB and inhibits their activities [12]. The GATOR1 complex is further regulated by its upstream inhibitor, the GATOR2 
complex, which consists of five subunits: Mios, WDR59, WDR24, Seh1L, and Sec13 [12].

Recently, the GATOR2 complex has been found to have a critical role in mediating amino acids signals, especially leucine and arginine, to the mTORC1 pathway. The sestrin protein was identified as a cytosolic leucine sensor, and interacts with GATOR2 to inhibit mTORC1 signaling when leucine is scarce [13-16]. On the other hand, the CASTOR1 homodimer and the CASTOR1-CASTOR2 heterodimer function as a cytosolic arginine sensor, and negatively regulate mTORC1 activity by binding to and inhibiting GATOR2 [17]. In the absence of arginine, CASTOR 1 binds to GATOR2 and inhibits mTORC1 signaling; whereas in the presence of arginine, CASTOR 1 interacts with arginine and no longer associates with GATOR2. Both CASTOR1 and CASTOR2 were found to contain two putative Aspartate kinase, Chorismate mutase, and TyrA (ACT) domains which function to recognize small ligands such as amino acids and nucleotides [18-24]. Besides Sestrin and CASTOR proteins, the membrane transporter SLC38A9 was reported to be a lysosomal arginine sensor, and transmit the arginine signal to $\mathrm{mTORC} 1$ by binding to the Ragulator complex [25-27].

In this study, we determined the crystal structure of human CASTOR1 in complex with a tightly bound arginine. Our structure shows that CASTOR1 dimerizes mainly through juxtaposition of its $\alpha 1$ and $\alpha 5$ helices. An exquisitely tailored pocket, carved between the $\mathrm{N}$-terminal domain (NTD) and the C-terminal domain (CTD) of CASTOR1, is employed to recognize arginine, and mutation of critical residues in this pocket abolished or diminished arginine-binding. Comparison with a prokaryotic aspartate kinase hinted that a surface patch on CASTOR1-NTD was involved in recognizing and activating GATOR2 (in particular, its subunit Mios only), which was confirmed by our pull-down assay using purified proteins. NM analysis suggested that a possible slicing motion between CASTOR1-NTD and -CTD might lead to exposure of its GATOR2-binding residues, thus regulating its association with GATOR2. Most likely, this breathing motion of CASTOR1 is correlated with arginine-binding. Finally, we proposed a model of how dimerization of CASTOR 1 relieves the inhibition of GATOR1 by GATOR2.

\section{Results}

\section{Overall structure of CASTORI}

By using the single-wavelength anomalous dispersion (SAD) method with a selenomethionine (SeMet) derivative, we determined the crystal structure of fulllength human CATSOR 1 in complex with arginine to $2.07 \AA$ resolution, with the $R_{\text {work }} / R_{\text {free }}$ factors being $19.00 \% / 23.83 \%$ (Table 1). In the crystal structure, CASTOR1 forms a dimer with the two protomers related by a two-fold rotation axis, and each protomer contains a bound arginine (Figure 1a). Dimerization buries $\sim 935 \AA^{2}$ of surface area for each protomer, and is mainly mediated through juxtaposition of the $\alpha 1$ and $\alpha 5$ helices of CASTOR1 (Figure 1b and c). Ile28 and Ile202 from both protomers pack together by hydrophobic interactions; and Trp21, Leu22, and Pro64 from both protomers also gather up to form the van der Waals interactions with each other. In addition, Tyr207 of one protomer donates a hydrogen bond to His 25 of the other protomer; and there exist salt bridges between Arg36 and Asp203 from different protomers. Consistent with our structural observation, the CASTOR1 protein in solution is a dimer, as assayed by the size exclusion chromatography-multiangle static light-scattering (SEC-MALS) method (Supplementary Figure S1). In contrast, mutation of L22R or I202D disrupted dimerization of CASTOR1 (Figure 1d).

Classical ACT domains possess a $\beta \alpha \beta \beta \alpha \beta$ topology $[18,19]$. Yet, our structure shows that each protomer of CASTOR1 actually contains four, rather than the proposed two [17], ACT domains. ACT1 (residues 1-75) and ACT2 (residues 76-153) assemble into the N-terminal domain (NTD, residues 1-153), while ACT3 (residues 175-260) and ACT4 (residues 261-329) form the C-terminal domain (CTD, residues 175-329). Both NTD and CTD have a $\beta \beta \alpha \beta \beta \alpha \beta \beta \alpha \beta \beta \alpha$ topology (there are two extra $\beta$-strands at the $\mathrm{C}$-terminal end of NTD) (Supplementary Figure S2) and highly resemble each other, with the root-mean-square deviation (RMSD) being $1.053 \AA$ for 80 aligned $\mathrm{C} \alpha$ atoms (Supplementary Figure S3). The NTD and CTD are reminiscent of two halves of a sphere, and the bound arginine molecule is encased inside this sphere (Figure 1e).

\section{The arginine-binding site of CASTOR1}

The arginine molecule is completely buried inside CASTOR1, and is located at the interface between CASTOR1-NTD and -CTD (Figure 2a and Supplementary Figure S4). Both NTD and CTD employ a surface pocket highly complementary to the shape of arginine (Figure 2b), and residues from both domains contribute to its specific recognition. The side-chain carboxyl group of Asp304 and the mainchain carbonyl groups of Gly274, Thr300, Phe301, 
Table 1 Data collection and refinement statistics

\begin{tabular}{|c|c|c|}
\hline & $\begin{array}{c}\text { SeMet- } \\
\text { CASTORI-arginine }\end{array}$ & $\begin{array}{c}\text { CASTOR1-arginine } \\
\text { complex }\end{array}$ \\
\hline \multicolumn{3}{|l|}{ Data collection } \\
\hline Space group & $\mathrm{C} 2$ & P21 \\
\hline \multicolumn{3}{|l|}{ Cell dimensions } \\
\hline $\mathrm{a}, \mathrm{b}, \mathrm{c}(\AA)$ & $95.86,77.50,48.09$ & $47.55,76.83,95.22$ \\
\hline$\alpha, \beta, \gamma\left(^{\circ}\right)$ & $90,94.02,90$ & $90,96.21,90$ \\
\hline Resolution $(\AA)$ & $50-1.96(2.03-1.96)$ & $50-2.07(2.14-2.07)$ \\
\hline$R_{\text {merge }}(\%)$ & $8.0(>100.0)$ & $10.0(99.4)$ \\
\hline $\mathrm{I} / \sigma \mathrm{I}$ & $11.3(1.0)$ & $11.5(1.2)$ \\
\hline $\mathrm{CC} 1 / 2$ & 0.524 & 0.583 \\
\hline Completeness (\%) & $99.8(99.4)$ & $99.7(99.3)$ \\
\hline Redundancy & $3.3(3.1)$ & $3.3(3.0)$ \\
\hline \multicolumn{3}{|l|}{ Refinement } \\
\hline Resolution (A) & & $50-2.07$ \\
\hline No. of reflections & & 31497 \\
\hline$R_{\mathrm{work}} / R_{\text {free }}$ & & $19.00 \% / 23.83 \%$ \\
\hline \multicolumn{3}{|l|}{ No. of atoms } \\
\hline Protein and ligand & & 4805 \\
\hline Ion & & 1 \\
\hline Solvent & & 149 \\
\hline \multicolumn{3}{|l|}{ B-factors $\left(\AA^{2}\right)$} \\
\hline Overall & & 32.12 \\
\hline Protein and ligand & & 32.11 \\
\hline Ion & & 10.64 \\
\hline Solvent & & 32.53 \\
\hline RMSD bond length $(\AA)$ & & 0.0167 \\
\hline RMSD bond angles $\left({ }^{\circ}\right)$ & & 1.8714 \\
\hline \multicolumn{3}{|l|}{ Ramachandran plot } \\
\hline Favored (\%) & & 92.6 \\
\hline Additional allowed (\%) & & 7.0 \\
\hline Generously allowed (\%) & & 0.2 \\
\hline Disallowed (\%) & & 0.2 \\
\hline
\end{tabular}

Abbreviations: ASU, asymmetric unit; r.m.s.d., root-mean-square deviations from ideal geometry.

$R_{\text {merge }}=\Sigma_{\mathrm{h}} \Sigma_{\mathrm{i}}\left|I_{\mathrm{h}, \mathrm{i}}-I_{\mathrm{h}}\right| / \Sigma_{\mathrm{h}} \Sigma_{\mathrm{i}} I_{\mathrm{h}, \mathrm{i}}$ for the intensity (I) of observation $\mathrm{i}$ of reflection h. $R$ factor $=\Sigma \| F_{\text {obs }}|-| F_{\text {calc }}|/| \Sigma\left|F_{\text {obs }}\right|$, where $F_{\text {obs }}$ and $F_{\text {calc }}$ are the observed and calculated structure factors, respectively. $R_{\text {free }}=R$ factor calculated using $5 \%$ of the reflection data chosen randomly and omitted from the start of refinement. Data for the highest-resolution shell are shown in parentheses.

and Phe303, all from CASTOR1-CTD, form chargestabilized hydrogen bonds with the guanidinium group of arginine (Figure 2c). In addition, the side-chain hydroxyl group of Ser111 and the main-chain carbonyl group of Val112, both from CASTOR1-NTD, accept hydrogen bonds from the main-chain amino group of arginine (Figure 2c). Besides, the main-chain amino group of Ile280 and the main-chain carbonyl group of Glu277, both from CASTOR1-CTD, make hydrogen bonds with the main-chain carboxyl group of arginine (Figure 2c).

To verify our structural observations, we performed point mutations on key residues in the arginine-binding pocket of CASTOR1, and carried out the isothermal titration calorimetry (ITC) assay to measure their binding affinities for arginine. In contrast to wild-type (WT) CASTOR 1 whose dissociation constant $\left(K_{\mathrm{d}}\right)$ for arginine was measured to be $2.23 \mu \mathrm{M}$ (Figure $2 \mathrm{~d}$ and Table 2), both double mutation of S111A/D304A (Supplementary Figure S5 and Table 2) and single mutation of D304A completely abrogated arginine binding (Figure $2 \mathrm{f}$ and Table 2), and single mutation of S111A resulted in an increase of $K_{\mathrm{d}}$ to $9.68 \mu \mathrm{M}$ (Figure $2 \mathrm{~g}$ and Table 2). Interestingly, WT CASTOR1 exhibited no detectable association with lysine (Figure 2e and Table 2), which among the twenty natural L-amino acids is the most similar one to arginine in terms of charge and size. Leucine, another amino acid that has an important role in the regulation of mTORC1 pathway[28-29], also has no measurable interaction with CASTOR1 (Supplementary Figure S6 and Table 2). This indicates that the sensing of arginine by CASTOR1 is highly specific and selective. The arginine-binding sites on both CASTOR1-NTD and -CTD are highly conserved, whereas the outside surface of CASTOR1 displayed no similar extent of conservation (Figure 2h).

\section{Arginine tethers the NTD and the CTD domains of CASTORl together}

As described above, the NTD and the CTD domains of CASTOR1 resemble two halves of a sphere. Their association results in closure of the sphere and encasement of the bound arginine. The NTD and the CTD interact through four layers of interfaces: the one close to NTD- $\beta 7$ and CTD- $\beta 17$, which also involves the contribution of the bound arginine (Figure $3 \mathrm{a}$, top left), the $\beta$-sheet formation between NTD- $\beta 3$ and CTD- $\beta 13$ (Figure 3a, top right), the pairing between two helices NTD- $\alpha 3$ and CTD- $\alpha 7$ (Figure 3a, bottom left), and the juxtaposition of NTD- $\alpha 1$ and CTD- $\alpha 5$ helices (Figure 3a, bottom right).

Compared with the second interface where the coupling between NTD- $\beta 3$ and CTD- $\beta 13$ results in the formation of seven hydrogen bonds, there are only two hydrogen bonds contributed by the pairing of NTD- $\beta 7$ and CTD- $\beta 17$ at the first interface, which is the most likely entrance for the arginine to squeeze into the CASTOR 1 molecule. The admission of arginine further tethers the NTD and the CTD domains of CASTOR 1 together by binding to residues from both the NTD (such as Ser111) and the CTD (such as Asp304). In addition, residues from the loop between $\beta 16$ and $\alpha 7$, which belongs to the CTD, make further contribution to the interaction between CASTOR1-NTD and -CTD. Asp276 forms 
a

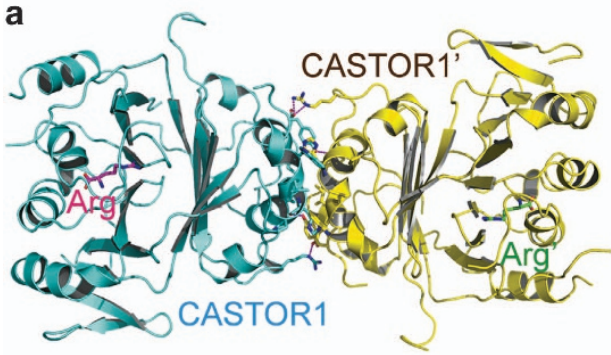

b
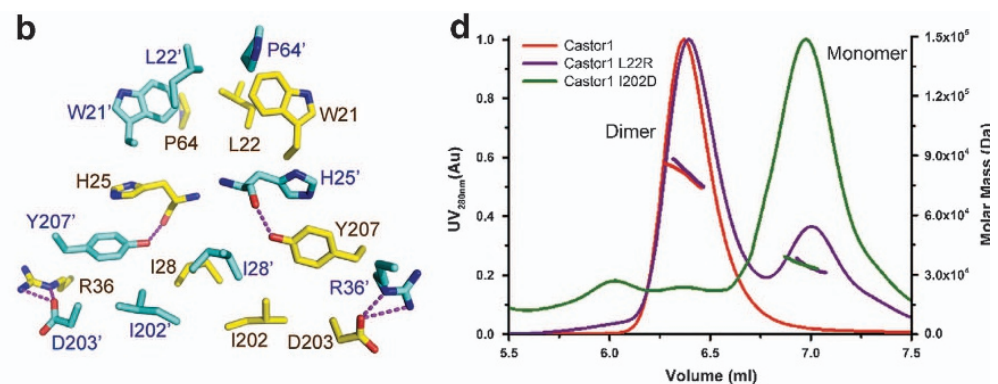

c

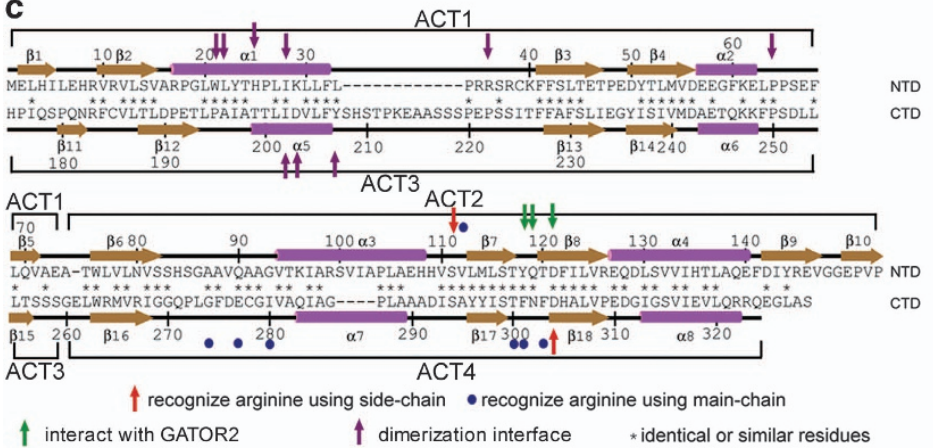

e

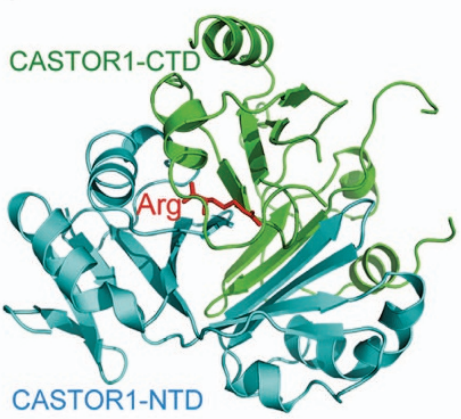

Figure 1 Structure of human CASTOR1. (a) Overall structure of the human CASTOR1 dimer. The two CASTOR1 protomers are colored in cyan and yellow, respectively; and the two bound arginine molecules are colored in magenta and green, respectively. (b) The dimerization interface of CASTOR1. The residues belonging to the two CASTOR1 protomers are colored in cyan and yellow, respectively. Hydrogen bonds are shown as magenta dashed lines. (c) Residues of the NTD and the CTD of CASTOR1 are aligned, with their secondary structures shown. Residues recognizing arginine using side-chains or main-chains are marked by red arrows or blue circles, respectively; while residues interacting with GATOR2 and mediating CASTOR1 dimerization are indicated by green arrows and purple arrows, respectively. (d) Mutation of L22R or I202D disrupted dimerization of CASTOR1, as assayed by the SEC-MALS experiment. (e) Structure of a protomer of CASTOR1. The NTD and the CTD domains of CASTOR1 are colored in cyan and green, respectively. The bound arginine is shown in red.

electrostatic interaction with Arg126 from the NTD, while Glu277 makes a charge-stabilized hydrogen bond with His175, which is close to the NTD. Besides, there is another hydrogen bond between Cys278 and the main-chain of Ser111 from the NTD (Figure 3a). Therefore, the first interface is the most probable site for arginine to enter CASTOR1, and, when bound, it strengthens the association between the NTD and the CTD which is further stabilized by the loop between $\beta 16$ and $\alpha 7$.

In agreement with our structural observation, the melting temperature $\left(T_{\mathrm{m}}\right)$ of CASTOR 1 in the presence and in the absence of arginine was measured to be $69.8^{\circ} \mathrm{C}$ and $65.7^{\circ} \mathrm{C}$, respectively, by the differential scanning calorimetry (DSC) assay (Figure 3b). Therefore, the enclosure of arginine causes the CASTOR 1 protein to fold more tightly, and leads to an almost $4{ }^{\circ} \mathrm{C}$ of increment in its $T_{\mathrm{m}}$. In contrast, CASTOR2, which did not have detectable binding affinity for arginine (Supplementary Figure S7), did not exhibit much change in $T_{\mathrm{m}}$ in the presence or absence of arginine (Figure 3c).
CASTOR1 employs a surface patch on its NTD to interact with the GATOR2 subunit Mios

As noted from its sequence homology [17], the structure of CASTOR 1 bears similarity to those of other ACT domain-containing proteins, especially the ACT domains of Escherichia coli aspartate kinase (PDB code: 2J0X) [30] and cyanobacteria aspartate kinase (PDB code: 3L76) [31]. Similar to CASTOR1, these prokaryotic aspartate kinases also contain bound amino acids. There are two bound lysine molecules for the ACT domain of E. coli aspartate kinase (Supplementary Figure S8a), while two lysines and two threonines are associated with the ACT domain of the cyanobacteria aspartate kinase (Supplementary Figure S8b). Their secondary structure organizations and binding sites for amino acids are all similar to those of CASTOR1 (Supplementary Figure S8c).

In the structure of $E$. coli aspartate kinase, there is an $\mathrm{N}$-terminal kinase domain (KD) and a C-terminal ACT domain. The ACT domain's interaction interface for the $\mathrm{KD}$ is on the opposite side to its lysine-recognition pocket (Figure 4a). Hence, the 
a

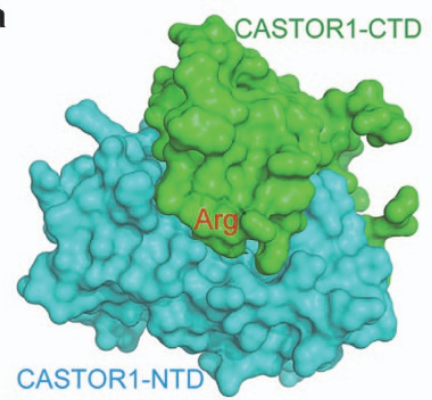

c

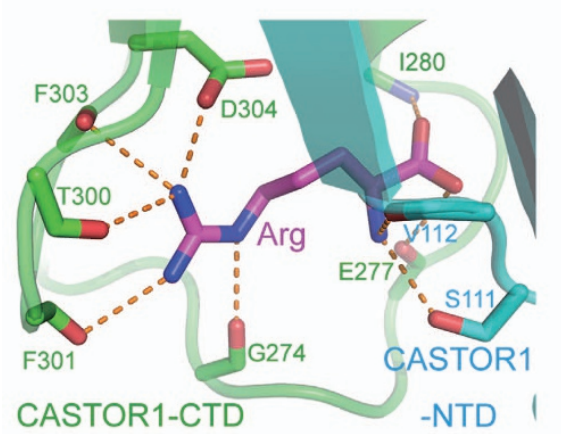

b
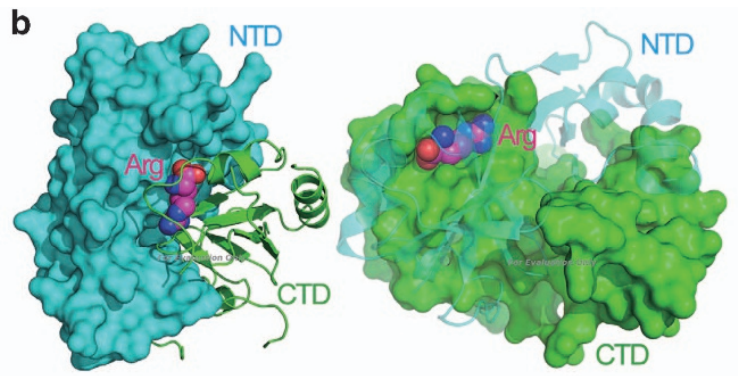

d

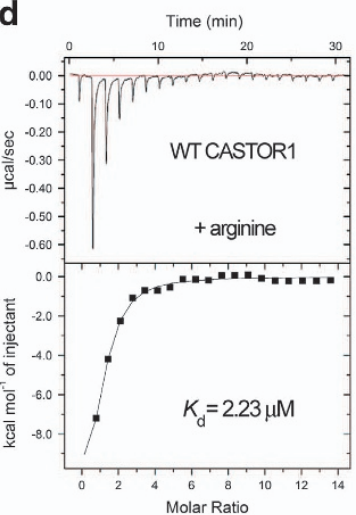

e

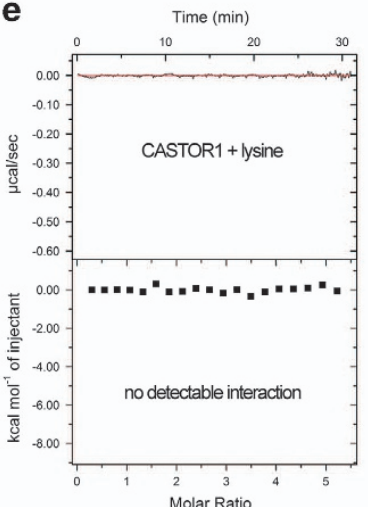

f

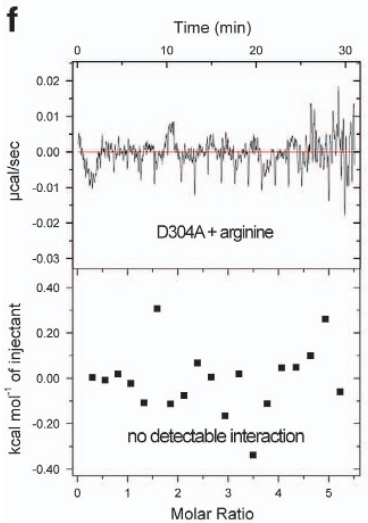

$\mathbf{g}$

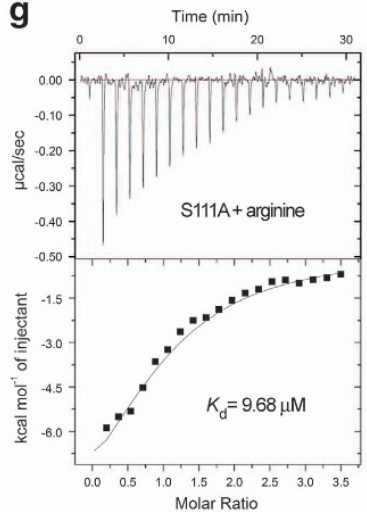

h

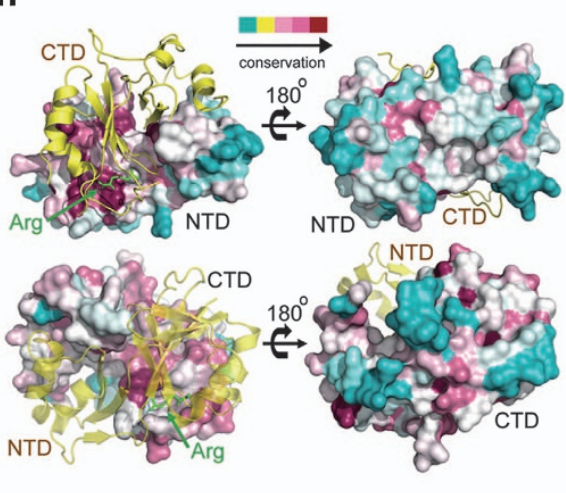

Figure 2 The arginine-binding pocket of CASTOR1. (a) The arginine is buried between the NTD and the CTD domains of CASTOR1. (b) Both the NTD and the CTD of CASTOR1 employ highly complementary surfaces to recognize the arginine, which is shown in sphere representation. (c) The arginine-binding pocket of CASTOR1. Residues from CASTOR1-NTD or CASTOR1-CTD are colored in cyan or green, respectively. The bound arginine is colored in magenta. Hydrogen bonds are represented as orange dashed lines. (d) The dissociation constant $\left(K_{\mathrm{d}}\right)$ between wild-type (WT) CASTOR1 and arginine was measured by ITC to be $2.23 \mu \mathrm{M}$. (e) No measurable interaction was detected between CASTOR1 and lysine. (f) Point mutation of Asp304 to alanine disrupted the association between CASTOR1 and arginine. (g) The S111A mutation diminished the binding affinity of CASTOR1 for arginine, with the $K_{d}$ value increasing to $9.68 \mu \mathrm{M}$. (h) The arginine-binding sites on CASTOR1-NTD and -CTD are highly conserved, whereas the outside surface of CASTOR1 is not conserved.

lysine serves as a ligand for the ACT domain which allosterically regulates its association with the KD. Through comparison, we hypothesized that the surface patch on CASTOR1-NTD involving Tyr118, Gln119, and Asp121, opposite to where its ligand arginine binds, might be the interface for association with its downstream effector, the GATOR2 complex
(Figure 4b). Indeed, triple mutation of Y118A/Q119A/ D121A on CASTOR1 pulled down much less purified GATOR2 protein complex (Figure 4c). Interestingly, mutation of $\mathrm{D} 304 \mathrm{~A}$ or S111A, which abrogated or decreased arginine binding (Figure $2 \mathrm{f}$ and $\mathrm{g}$ ), enhanced the amount of GATOR2 complex pulled down by CASTOR1 (Figure 4c). 
Table 2 Dissociation constants $\left(K_{\mathrm{d}}\right)$ for the interaction between various CASTOR1/CASTOR2 constructs and various amino acids as measured by the isothermal titration calorimetry assay

\begin{tabular}{llcccc}
\hline CASTORI/CASTOR2 & Amino acid & Molar ratio & $K_{d}(\mu \mathrm{M})$ & $\Delta H\left(\mathrm{kcal} \mathrm{mol}^{-1}\right)$ & $\left.\mathrm{T \Delta S}_{(\mathrm{kcal} \mathrm{mol}}^{-1}\right)$ \\
\hline WT CASTOR1 & Arginine & $1.01 \pm 0.10$ & $2.23 \pm 0.20$ & $-13.4 \pm 1.7$ & -6.05 \\
WT CASTOR1 & Lysine & ND & ND & ND & ND \\
WT CASTOR1 & Leucine & ND & ND & ND & ND \\
WT CASTOR2 & Arginine & ND & ND & ND & ND \\
CASTOR1-S111A & Arginine & $0.95 \pm 0.09$ & $9.68 \pm 3.20$ & $-1.81 \pm 0.24$ & 5.19 \\
CASTOR1-D304A & Arginine & ND & ND & ND & ND \\
S111A/D304A & Arginine & ND & ND & ND & ND \\
\hline
\end{tabular}

Abbreviation: ND, no detectable interaction was observed.

\pm values, errors of curve fitting.

Mios, one out of the five subunits of GATOR2, was reported to participate in the association with CASTOR 1 [17]. We employed the $\mathrm{Ni}^{2+}$-NTA pull-down assay to examine the interaction between each of the five subunits of GATOR2 and CASTOR1 using purified proteins, and found that among the five subunits of GATOR2, only Mios exhibited considerable direct physical interaction with CASTOR1 (Figure 4d). In addition, by using the $\mathrm{Ni}^{2+}$-NTA pull-down assay with purified CASTOR1 and Mios proteins, we found that the Y118A/Q119A/D121A mutation, as compared with the WT control, caused substantial dissociation of Mios from CASTOR1 (Figure 4e). This is also the first experimental evidence, to our knowledge, that there exists direct interaction between CASTOR1 and Mios proteins. Furthermore, the Y118A/Q119A/D121A mutation disabled CASTOR1's ability to inhibit the downstream mTORC1 signaling, such as S6K1 phosphorylation, even in the absence of arginine (Figure 4f).

\section{Normal mode analysis suggests an 'open'-to-'closed' conformational switch for CASTORI}

To investigate potential conformational rearrangement of CASTOR1, we subjected our structure to the $\mathrm{NM}$ analysis [32]. Interestingly, the NM analysis revealed a breathing motion between the NTD and the CTD domains of CASTOR1. In the 'closed and compact' state which resembles more closely to the crystal structure, the NTD and the CTD domains of CASTOR1 are compressed against each other tightly. As a result, the GATOR2-binding residues Y118/ Q119/D121 are mostly buried and not available for contact with GATOR2 (Figure 5a and d). In contrast, in the 'open and relaxed' state, the NTD and the CTD domains of CASTOR 1 are much more separated and the GATOR2-binding surface patch is exposed, ready for interaction with GATOR2 (Figure $5 b$ and e).
Presumably, this conformational switch (Figure 5c and $f$ and Supplementary Video S1) of CASTOR1 is correlated with arginine-binding. In the absence of arginine, CASTOR 1 exists in the 'open' state. Its GATOR2-binding surface patch is exposed and associates with GATOR2. On the other hand, when arginine is present, the binding of arginine tethers the NTD and the CTD domains of CASTOR1 tightly together. Thus a conformational rearrangement of CASTOR 1 from the 'open' state to the 'closed' state is induced, and the GATOR2-binding surface patch of CASTOR 1 is concealed. Hence GATOR2 is detached from CASTOR 1 and is free to activate the downstream mTORC1 signaling.

\section{Discussion}

Arginine has a critical role in the regulation of many aspects of mammalian physiology, such as insulin secretion, intestinal cell migration, immune responses, and so on [33-35]. It is an interesting question of how cells sense the presence of arginine and transmit its signal. Our structure of the CASTOR1-arginine complex reveals that a nicely tailored pocket on CASTOR 1 is employed to recognize arginine, but not other common amino acids such as lysine and leucine (Figure 2). Furthermore, our NM analysis reveals that there is an intrinsic flexibility between the two domains of CASTOR1, and the relative movement between its two domains underlies its association/dissociation with its downstream effector GATOR2 (Figure 5), which is most likely regulated by arginine binding. This kind of intrinsic inter-domain flexibility and conformational switching regulated by a binding partner is reminiscent of other key mediators in important signal transduction pathways [36]. It will be interesting to elucidate how 


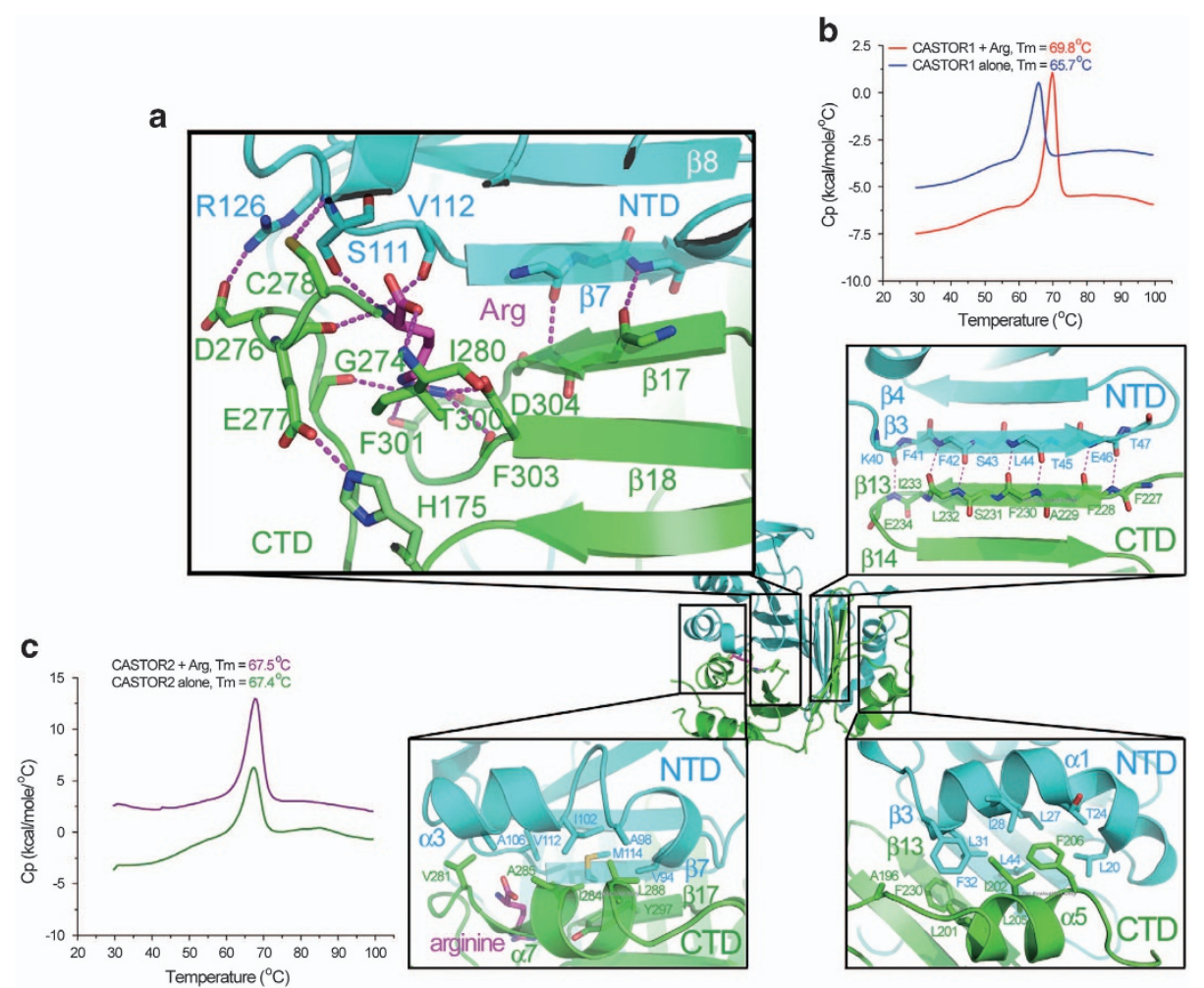

Figure 3 Arginine tethers CASTOR1-NTD and CASTOR1-CTD tightly together. (a) Arginine interacts with both the NTD and the CTD of CASTOR1, thus tethering the NTD and the CTD domains tightly together. The other three interfaces between CASTOR1-NTD and -CTD are also shown. (b) The DSC assay demonstrated that arginine promoted tighter folding of CASTOR1, as shown by the increase of its melting temperature $\left(T_{\mathrm{m}}\right)$. (c) The DSC assay revealed that the melting temperature of CASTOR2 was not affected by the addition of arginine.

exactly arginine binding induces the inter-domain dynamic movement of CASTOR1.

Human CASTOR2 is a close homolog of CASTOR 1, but it does not recognize arginine (Table 2) and it is constitutively associated with GATOR2. Our DSC assay suggests that it folds tighter than CASTOR1 (Supplementary Figure S9). Comparison of primary sequences of CASTOR1 orthologues and CASTOR2 orthologues suggested that residues at positions of 108-110 and 302 (CASTOR 1 residue numbers; H108/H109/V110 and N302 for CASTOR1, while Q110/N111/I112 and K303 for CASTOR2) might be responsible for their different argininebinding abilities (Supplementary Figure S10). Occurrence of amimo acids different from CASTOR 1 at these positions of CASTOR2 might underlie its incapability to arginine, which is consistent with the findings of other groups [37-38]. Regrettably, we were not able to obtain diffracting crystals of CASTOR2 despite considerable efforts. It remains an important question of how the structure of CASTOR2 differs from CASTOR1. This structural information might provide an answer to why it does not interact with arginine but associates with GATOR 2 constitutively.

The GATOR2 complex, which is the downstream effector of CASTOR1, consists of five subunits, Mios, WDR59, WDR24, Seh1L, and Sec13 [12]. In a previous report, WDR24, Mios, and Seh1L have been implicated in CASTOR1 binding [17]. However, it is still unknown which subunit(s) provides direct physical contact with CASTOR1. Using purified proteins, we found that among the five subunits of GATOR2, only Mios directly interacts with CASTOR1. It would be interesting to further examine the structural mechanism of how Mios associates with CASTOR1.

In the mean time when this manuscript was being prepared, reports on the crystal structure of CASTOR 1 in complex with arginine were published by the Sabatini and co-workers [37], as well as by the Ding and co-workers [38]. Their reports obtained similar structural observations as ours, both on the overall structure and the arginine-binding pocket. In Xia et al. [38], the dissociation constant $K_{\mathrm{d}}$ between CASTOR 1 and arginine was measured by ITC to be $5.5 \mu \mathrm{M}$, 

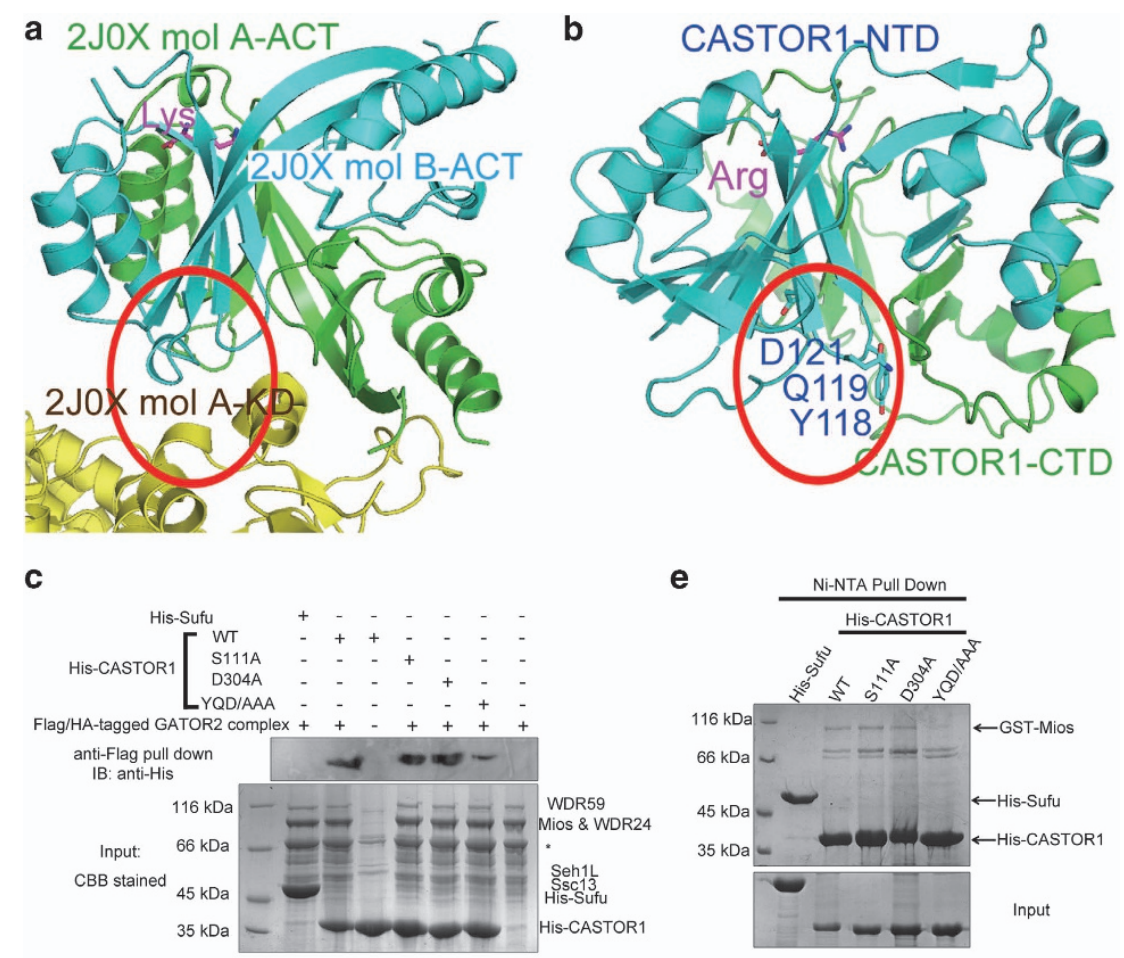

d

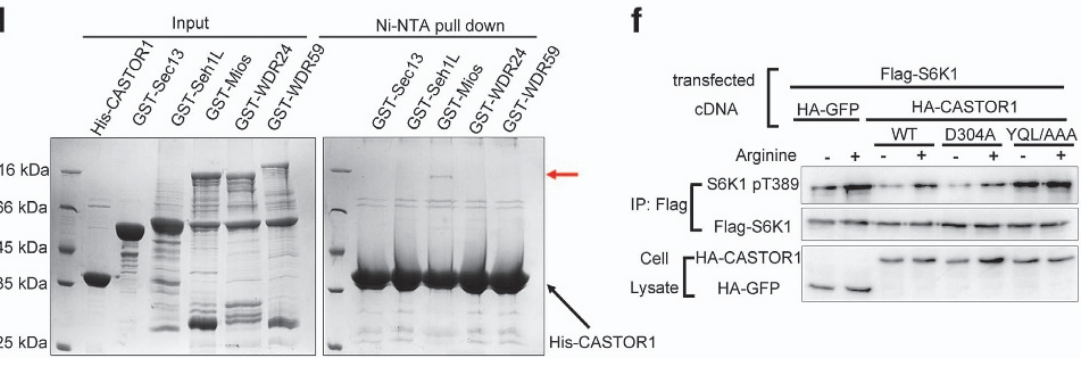

Figure 4 A surface patch on CASTOR1-NTD on the opposite side of the arginine-binding site mediates its association with GATOR2. (a) The ACT domain of E. coli aspartate kinase (PDB code: 2JOX) employs a surface patch on the opposite side of its lysine-binding site to associate with its KD. In this structure, E. coli aspartate kinase exists as a dimer. The two protomers are denoted as mol A and mol B, respectively. (b) A surface patch on CASTOR1-NTD at a similar position as the E. coli aspartate kinase ACT domain's binding site for its KD domain was identified to mediate its association with GATOR2. (c) Mutation of Y118A/Q119A/D121A on CASTOR1 decreased its interaction with the purified GATOR2 complex. His-tagged Suppressor of Fused (Sufu) protein served as a control. (d) Out of the five subunits of GATOR2, only Mios exhibited direct physical interaction with CASTOR1, as assayed by the $\mathrm{Ni}^{2+}$-NTA pull-down experiment using purified proteins. (e) Mutation of Y118A/Q119A/D121A on CASTOR1 almost abrogated its interaction with the purified Mios protein, a component of the GATOR2 complex. His-tagged Sufu served as a control. (f) Mutation of Y118A/Q119A/D121A abolished CASTOR1's ability to inhibit GATOR2, and the downstream mTORC1 kinase phosphorylated S6K1 even in the absence of arginine.

which is similar to our result. On the other hand, in Chantranupong et al. [17], the $K_{\mathrm{d}}$ value was found to be $34.8 \mu \mathrm{M}$, as assayed through measuring the amount of $\left[{ }^{3} \mathrm{H}\right]$ radio-labeled arginine pulled down by immunoprecipitated CASTOR1. This discrepancy might be caused by the difference between in vivo versus in vitro assay methods. Within cells, there might be other arginine-binding proteins other than CASTOR1, which would compete with it for arginine binding and decrease the effective binding affinity between CASTOR 1 and arginine. In Saxton et al. [37], they also revealed that Y118/Q119/D121 of CASTOR1 were involved in GATOR2-binding and mutation of Y118/Q119/D121 disrupted CASTOR1-binding to GATOR2 and activated downstream S6K1 phosphorylation in the absence of arginine. However, their in vivo co-IP approach could not exclude the possibility that some other protein(s) might mediate 

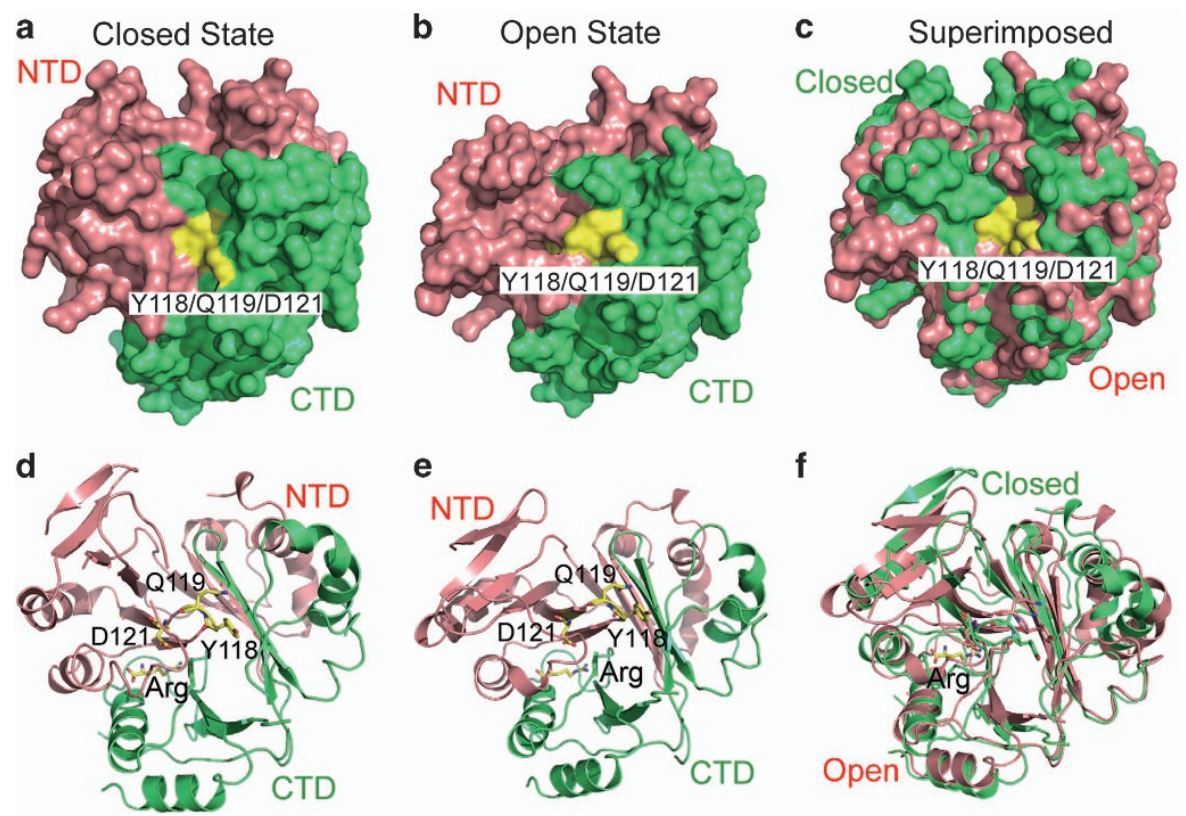

Figure 5 Normal mode analysis suggests that there exists an 'open'-to-'closed' conformational switch for CASTOR1. (a, d) In the 'closed' state of CASTOR1 which resembles more closely to the crystal structure, the NTD and the CTD domains are more compressed against each other, and the GATOR2-binding residues are mostly buried. (b, e) In the 'open' state of CASTOR1, the NTD and the CTD domains are more separated, and the GATOR2-binding residues are exposed. (c, $\mathbf{f}$ ) Superimposition of the 'closed' and the 'open' states of CASTOR1. (a-c) Surface representations. (d-f) Cartoon representations.

the interaction between CASTOR1 and Mios. Our in vitro pull-down result using purified proteins is complementary to their finding, and provides direct evidence for physical interaction between residues Y118/Q119/D121 of CASTOR1 and Mios, but not the other four subunits of GATOR2.

Both CASTOR 1 and CASTOR2 function as dimers to relieve the inhibition of GATOR1 by GATOR2 [17], so that GATOR1 can prevent the RagA/RagB RagC/RagD complex from activating mTORC1. Interestingly, it was reported that dimerizationdeficient CASTOR1 mutant displayed weaker interaction with GATOR2 and failed to inhibit downstream mTORC1 signaling [37]. Examination of the structure of CASTOR1 dimer revealed that the GATOR2binding sites (that is, residues Y118/Q119/D121) of both CASTOR 1 protomers reside on the same side and face the same direction (Figure 6a). This inspired us to propose a model of how dimerization of CASTOR1 (and maybe CASTOR2 as well) relieves the inhibition of GATOR 1 by GATOR 2 (Figure $6 \mathrm{~b}$ ). In the presence of arginine, GATOR2 dissociates from CASTOR1, and binds to GATOR 1 to inhibit its GAP activity towards RagA/RagB. GTP-bound $\operatorname{RagA} / \operatorname{RagB}$ in complex with GDP-bound $\mathrm{RagC} / \mathrm{RagD}$ activates mTORC1 (Figure 6b, left). On the other hand, when arginine is absent, the GATOR2-binding site on
CASTOR 1 is exposed, and each of the CASTOR 1 protomer is bound by a GATOR2 complex. Juxtaposition of two gigantic GATOR2 complexes ( $\sim 378 \mathrm{kDa}$ for a GATOR2 complex) on the relatively small CASTOR 1 dimer $(\sim 36 \mathrm{kDa}$ for a CASTOR 1 momomer) creates severe spatial hindrance for the two GATOR1 complexes associated with GATOR2, which are also huge $(\sim 285 \mathrm{kDa}$ for a GATOR 1 complex). Therefore, steric clash would force GATOR1 to dissociate from GATOR2, hence stimulating the hydrolysis of GTP bound with RagA/RagB and inhibiting the downstream mTORC1 activity (Figure 6b, right). Interestingly, to our knowledge, similar dimeric assembly as that of CASTOR1 is not found in bacterial proteins possessing ACT domains such as aspartate kinases. We hypothesize that the dimeric assembly of CASTOR proteins specifically evolves to engage the GATOR2/GATOR1 system, which is present in metazoa but not in prokaryotes.

\section{Methods}

\section{Protein expression and purification}

Full-length human CASTOR1 and CASTOR2 were subcloned into the pET28a vector, with an N-terminal $6 \times$ His-tag. The cDNA encoding full-length human Mios was subcloned 
10

a

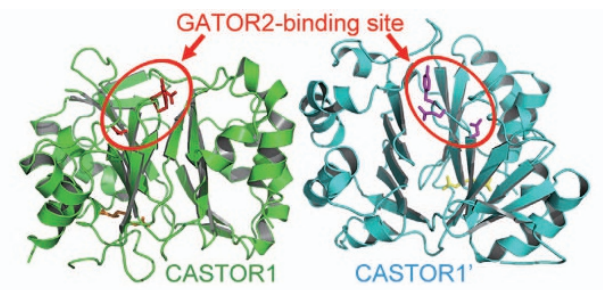

b

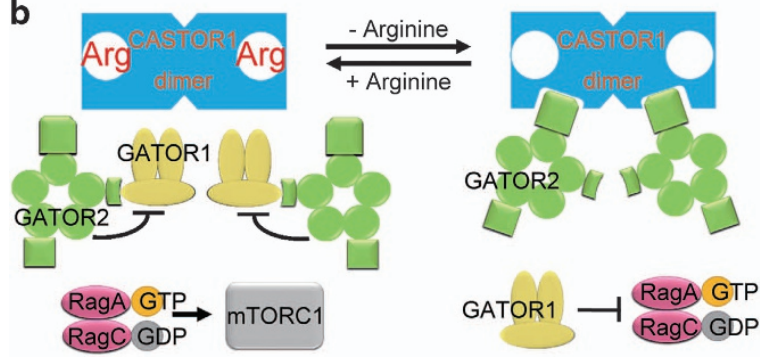

Figure 6 Dimerization of CASTOR1 might have a critical role in relieving the inhibition of GATOR1 by GATOR2. (a) In the structure of CASTOR1 dimer, the GATOR2-binding sites (residues Y118/Q119/D121, marked by red circles) on the two CASTOR1 protomers are located on the same side and face the same direction. (b) A schematic model showing that the dimerization of CASTOR1 leads to juxtaposition of two GATOR2 complexes on the relatively small CASTOR1 dimer, which creates spatial hindrance for their bound GATOR1 complexes. GATOR1 is thus dissociated from GATOR2 and relieved from its inhibition.

into a pGEX4T-1 vector, with an N-terminal GST tag. These vectors were transformed into $E$. coli BL21(DE3)-competent cells. Transformed cells were cultured at $37{ }^{\circ} \mathrm{C}$ until the optical density (OD) at $600 \mathrm{~nm}$ was 0.8 , and protein expression was induced with $0.2 \mathrm{~mm}$ IPTG for $14-18 \mathrm{~h}$ at $16^{\circ} \mathrm{C}$. After harvesting and resuspension, cells were lysed with a cell homogenizer (JNBIO, Guangzhou, China). The lysate was cleared by centrifugation at 14000 r.p.m. for $30 \mathrm{~min}$ and the supernatants were subjected to $\mathrm{Ni}^{2+}$-NTA or GST affinity chromatography (GE healthcare, Little Chalfont, UK). The eluted protein was loaded onto a Superdex 200 gel filtration column (GE Healthcare) in $15 \mathrm{~mm}$ Tris- $\mathrm{HCl}, \mathrm{pH} 8.0,150 \mathrm{~mm} \mathrm{NaCl}$, and $3 \mathrm{~mm}$ dithiothreitol (DTT). Peak fractions were combined, supplemented with $2 \mathrm{~mm}$ arginine, and incubated for $30 \mathrm{~min}$ before being concentrated to $10 \mathrm{mg} \mathrm{ml}^{-1}$ for crystallization. Constructs of CASTOR 1 mutants were derived by the whole plasmid PCR/ DpnI digestion method, and were verified by sequencing. All the mutant CASTOR1 proteins were purified using the same procedure as for the WT proteins. Selenomethionine (SeMet)-derivatized CASTOR1 was expressed using the methionineautotrophic E. coli B834(DE3) cells cultured in M9 media and purified as the native protein, except that $5 \mathrm{~mm}$ DTT was used in the gel filtration buffer.

\section{Crystallization, data collection, and structure determination}

Native and SeMet-substituted human CASTOR1 protein crystals were grown at $14^{\circ} \mathrm{C}$ by the hanging-drop vapordiffusion method, with $1 \mu \mathrm{l}$ of protein mixed with an equal volume of reservoir solution containing 18\% PEG 3350 and $0.2 \mathrm{M}$ magnesium acetate. Crystals were cryoprotected in the crystallization buffer supplemented with $25 \%$ glycerol. One set of single-wavelength anomalous dispersion (SAD) data of SeMet-CASTOR 1 at $1.96 \AA$ resolution was collected at the beamline BL18U1, and one set of data of native CASTOR1 at $2.07 \AA$ resolution was collected at the beamline BL19U1 of National Center for Protein Sciences Shanghai at $100 \mathrm{~K}$. Data reduction was performed with the HKL3000 software [39]. The SAD phases for the SeMet-CASTOR1 were determined using the Autosol module of PHENIX [40], and the phases for the native CASTOR1 were determined using the Phaser [41] program in the CCP4 package [42] After automatic modelbuilding using the AutoBuild module of PHENIX [40] and manual model-building in Coot [43], refinement was performed by the REFMAC5 program [44] in the CCP4 package [42]. The crystals belonged to the space group $P 2{ }_{1}$, and contained two molecules of CASTOR1 in one asymmetric unit. The final refined model has $R_{\text {work }} / R_{\text {free }}$ factors of $19.00 \% / 23.83 \%$. The quality of the structural model was checked with the CCP4 program PROCHECK [42], which shows a good stereochemistry according to the Ramachandran plot.

\section{Isothermal titration calorimetry (ITC) and differential scanning calorimetry (DSC) assay}

ITC experiments were performed with an ITC200 system (MicroCal, Worcestershire, UK) at $25^{\circ} \mathrm{C}$ as described before [45]. Both ITC and DSC reaction buffers contained $25 \mathrm{~mm}$ HEPES-Na, pH 7.5, and $150 \mathrm{~mm} \mathrm{NaCl}$. All samples and buffer were centrifuged and degassed before experiments. To determine the $T_{\mathrm{m}}$ values of CASTOR 1 and CASTOR2 protein, VP-DSC (MicroCal) was used to measure the excess heat capacity of protein unfolding. For DSC analysis, protein samples at 1-2 mg/ $\mathrm{ml}$ were loaded into the sample cell and the reaction buffer was taken as the reference. The heat capacity change was measured from 30 to $100{ }^{\circ} \mathrm{C}$ with a scan rate of $90^{\circ} \mathrm{C} / \mathrm{h}$ and system pressure $\geq 35$ p.s.i. Buffer baseline scans were established before analysis of each protein sample. Data were analyzed using the Origin 7.0 software. Sample scans were buffer-subtracted and concentration-normalized.

\section{Cell culture, transfection, and plasmids}

Expi293F cells (Thermo Fisher Scientific, Waltham, MA, USA) were cultured in the Expi293 Expression medium (Thermo Fisher Scientific) on a shaker with $5 \% \mathrm{CO}_{2}$ at $37^{\circ} \mathrm{C}$. Cell transfection was performed using ExpiFectamine293 Transfection Kit (Thermo Fisher Scientific) as described in the manual. The pRK5 plasmids expressing Flag-WDR24, Flag-Mios, HA-WDR59, HA-Sec13, and HA-Seh1L were obtained from Addgene (Cambridge, MA, USA). The cDNAs encoding human CASTOR1 and human CASTOR2 were chemically synthesized (Union-Biotech, Shanghai, China).

\section{In vitro CASTOR1-GATOR2 dissociation assay}

Expi293F cells were harvested $48 \mathrm{~h}$ after co-transfection of the five subunits of GATOR2, and cells were undergone three cycles of freeze-and-thaw in the lysis buffer $(50 \mathrm{~mm}$ Tris- $\mathrm{HCl}$, $\mathrm{pH} 8.0,150 \mathrm{~mm} \mathrm{NaCl}$, supplemented with the protease inhibitor cocktail). After centrifugation at 14000 r.p.m. for 
$50 \mathrm{~min}$ at $4{ }^{\circ} \mathrm{C}$, the supernatants were subjected to affinity chromatography using the anti-FLAG M2 resin (Sigma, St Louis, MO, USA). After incubation at $4{ }^{\circ} \mathrm{C}$ for $1 \mathrm{~h}$, the resin was washed thoroughly with the lysis buffer, and $200 \mu \mathrm{g} \mathrm{ml}^{-1}$ FLAG peptide (with a sequence of DYKDDDDK) was used to elute bound proteins. After SDS-PAGE analysis, the eluted protein was subjected to the Superdex 200 gel filtration chromatography to remove the FLAG peptide.

The purified GATOR2 protein complex was mixed with His-tagged WT or mutant CASTOR1 protein with a molar ratio of $1: 3$. After $30 \mathrm{~min}$ of incubation at $4{ }^{\circ} \mathrm{C}$, the mixture was loaded onto an anti-FLAG affinity column. Bound proteins were eluted with buffer containing the FLAG peptide, and anti-His immunoblot was carried out to detect the bound CASTOR 1 pulled down by the GATOR 2 complex. His-tagged Suppressor of Fused (Sufu) protein was used as a control.

Binding assays between purified WT or mutant CASTOR1 proteins and WT GST-Mios were performed by the $\mathrm{Ni}^{2+}$-NTA column pull-down assay. The GST-Mios protein was added to His-CASTOR1 protein pre-immobilized on the $\mathrm{Ni}^{2+}$-NTA affinity column (GE Healthcare) at $4{ }^{\circ} \mathrm{C}$, washed with the $\mathrm{Ni}^{2+}$ -NTA column buffer ( $25 \mathrm{~mm}$ Tris, $\mathrm{pH} 8.0,300 \mathrm{~mm} \mathrm{NaCl}$, and $20 \mathrm{~mm}$ imidazole) and eluted with a buffer containing $25 \mathrm{~mm}$ Tris, $\mathrm{pH} 8.0,300 \mathrm{~mm} \mathrm{NaCl}$, and $500 \mathrm{~mm}$ imidazole. Eluted protein fractions were analyzed by SDS-PAGE and Coomassie Blue staining.

\section{Normal mode analysis}

The NM analysis was performed as described before [36, 46]. Briefly, the structural coordinates of CASTOR1 were submitted for the NM analysis using the Elastic Network Model server (http://www.igs.cnrsmrs.fr/elnemo/) [32], which is a fast and simple tool to compute the major (that is, low-frequency) NMs of a protein. The first vibrational mode (that is, the seventh $\mathrm{NM}$, the first six modes being translational and rotational motions of the system as a whole) generated by the server, which showed the clearest inter-domain movement between NTD and CTD and was correlated with the burying and exposing of the GATOR2-binding residues, was selected for further analysis.

\section{Size exclusion chromatography - multi-angle static}

light-scattering (SEC-MALS) assay

SEC-MALS experiments were carried out at $25^{\circ} \mathrm{C}$ with a DAWN HELEOS II 18-angle light-scattering instrument (Wyatt Technology, Santa Barbara, CA, USA) and a size exclusion chromatography WTC-015S5 column (Wyatt Technology) coupled to an Agilent 1100 HPLC system (Agilent, Santa Clara, CA, USA). Approximately $400 \mu \mathrm{g}$ protein was injected per run, in a buffer containing $15 \mathrm{~mm}$ Tris- $\mathrm{HCl}$, $\mathrm{pH} 8.0,150 \mathrm{~mm} \mathrm{NaCl}$, and $2 \mathrm{~mm}$ DTT. The molecular weight of each sample was calculated by the ASTRA 6.1 software (Wyatt Technology).

\section{S6K1 phosphorylation assay}

About 0.8 million HEK-293 T cells were plated in a 6-cm cell culture dish. After $24 \mathrm{~h}, 5 \mu \mathrm{g}$ HA-CASTOR1 or HA-GFP and Flag-S6K 1 constructs were co-transfected. $36 \mathrm{~h}$ later, cells were starved of arginine for $45 \mathrm{~min}$ with arginine-free DMEM medium or starved for $45 \mathrm{~min}$ and restimulated with $1 \mathrm{mM}$ arginine in cell media for $15 \mathrm{~min}$. Cells were rinsed with chilled PBS and lysed with Triton lysis buffer (1\% Triton X-100, $25 \mathrm{~mm}$ Tris- $\mathrm{HCl} \mathrm{pH} 7.4,100 \mathrm{~mm} \mathrm{NaCl}, 10 \mathrm{~mm} \mathrm{KCl}$, and $2.5 \mathrm{~mm}$ $\mathrm{MgCl}_{2}$ ) supplemented with EDTA-free protease inhibitor (Roche, Basel, Switzerland). The soluble lysate fractions were isolated by centrifugation at 14000 r.p.m. for $15 \mathrm{~min}$ at $4{ }^{\circ} \mathrm{C}$. The FLAG-M2 resin (Sigma) was added and incubated with the cell lysate for $4-6 \mathrm{~h}$ at $4{ }^{\circ} \mathrm{C}$. The beads were washed five times with the lysis buffer and boiled with the sample buffer for $5 \mathrm{~min}$. Samples were resolved by $12 \%$ SDS-PAGE and analyzed by immunoblot. Phospho-S6K1 (Thr389) antibody (Cell Signaling Technology, Beverly, MA, USA) was employed to detect phospho-S6K1.

\section{Molecular graphics}

All protein structure figures were generated by the PyMOL program [47]. Sequence conservation of CASTOR1 mapped onto the surface of its crystal structure was generated by the ConSurf server (http://consurf.tau.ac.il) [48].

\section{Accession codes}

The atomic coordinates and structure factors of the complex between full-length human CASTOR 1 and arginine have been deposited in the Protein Data Bank with the accession number $5 \mathrm{GV} 2$.

\section{Conflict of Interest}

The authors declare no conflict of interest.

\section{Acknowledgements}

We thank Hua Li, Zhenyi Zhang, and Liqiong Liu for their contributions to this work. We thank Wenming Qin, Lijie Wu, Huan Zhou, Jianhua He, and other staff at the BL19U1 and BL18U1 beamlines at National Center for Protein Sciences Shanghai (NCPSS), and at the BL17U1 beamline at Shanghai Synchrotron Radiation Facility. This work was supported by grants from the National Natural Science Foundation of China (grant numbers 31230002, 31470223, and 31670106), State Key Laboratory of Microbial Resources, Institute of Microbiology, Chinese Academy of Sciences, and the Program for Professor of Special Appointment (Eastern Scholar) at Shanghai Institutions of Higher Learning.

\section{Author contributions}

Protein purification, crystallization and data collection, normal mode analysis, $\mathrm{Ni}^{2+}$ column pull-down assay, and ITC assay were performed by ZG. SEC-MALS assay was performed by QW and WD. CY and LW assisted with protein purification. Crystal structure determination was performed by GW. ZG and GW designed the experiments and wrote the paper. 


\section{References}

1 Wullschleger S, Loewith R, Hall MN. TOR signaling in growth and metabolism. Cell 2006; 124: 471-484.

2 Laplante M, Sabatini DM. mTOR signaling in growth control and disease. Cell 2012; 149: 274-293.

3 Dibble CC, Manning BD. Signal integration by mTORC1 coordinates nutrient input with biosynthetic output. Nat Cell Biol 2013; 15: 555-564.

4 Jewell JL, Russell RC, Guan KL. Amino acid signalling upstream of mTOR. Nat Rev Mol Cell Biol 2013; 14: 133-139.

5 Sancak Y, Peterson TR, Shaul YD et al. The Rag GTPases bind raptor and mediate amino acid signaling to mTORC1. Science 2008; 320: 1496-1501.

6 Sancak Y, Bar-Peled L, Zoncu R, Markhard AL, Nada S, Sabatini DM. Ragulator-Rag complex targets mTORC1 to the lysosomal surface and is necessary for its activation by amino acids. Cell 2010; 141: 290-303.

7 Bar-Peled L, Schweitzer LD, Zoncu R, Sabatini DM. Ragulator is a GEF for the Rag GTPases that signal amino acid levels to mTORC1. Cell 2012; 150: 1196-1208.

8 Dibble CC, Elis W, Menon S et al. TBC1D7 is a third subunit of the TSC1-TSC2 complex upstream of mTORC1. Mol Cell 2012; 47: 535-546.

9 Menon S, Dibble CC, Talbott G et al. Spatial control of the TSC complex integrates insulin and nutrient regulation of mTORC1 at the lysosome. Cell 2014; 156: 771-785.

10 Tsun Z-Y, Bar-Peled L, Chantranupong L et al. The folliculin tumor suppressor is a GAP for the $\mathrm{RagC/D}$ GTPases that signal amino acid levels to mTORC1. Mol Cell 2013; 52: 495-505.

11 Petit CS, Roczniak-Ferguson A, Ferguson SM. Recruitment of folliculin to lysosomes supports the amino acid-dependent activation of Rag GTPases. J Cell Biol 2013; 202: 1107-1122.

12 Bar-Peled L, Chantranupong L, Cherniack AD et al. A tumor suppressor complex with GAP activity for the Rag GTPases that signal amino acid sufficiency to mTORC1. Science 2013; 340: 1100-1106.

13 Chantranupong L, Wolfson RL, Orozco JM et al. The sestrins interact with GATOR2 to negatively regulate the amino-acid-sensing pathway upstream of mTORC1. Cell Rep 2014; 9: 1-8.

14 Parmigiani A, Nourbakhsh A, Ding B et al. Sestrins inhibit mTORC1 kinase activation through the GATOR complex. Cell Rep 2014; 9: 1281-1291.

15 Wolfson RL, Chantranupong L, Saxton RA et al. Sestrin2 is a leucine sensor for the mTORC1 pathway. Science 2016; 351: $43-48$.

16 Saxton RA, Knockenhauer KE, Wolfson RL et al. Structural basis for leucine sensing by the Sestrin2mTORC1 pathway. Science 2016; 351: 53-58.

17 Chantranupong L, Scaria SM, Saxton RA et al. The CASTOR proteins are arginine sensors for the mTORC1 pathway. Cell 2016; 165: 153-164.

18 Grant GA. The ACT domain: a small molecule binding domain and its role as a common regulatory element. $J$ Biol Chem 2006; 281: 33825-33829.
19 Chipman DM, Shaanan B. The ACT domain family. Curr Opin Struct Biol 2001; 11: 694-700.

20 Siltberg-Liberles J, Martinez A. Searching distant homologs of the regulatory ACT domain in phenylalanine hydroxylase. Amino Acids 2009; 36: 235-249.

21 Tan K, Li H, Zhang R, Gu M, Clancy ST, Joachimiak A. Structures of open $(R)$ and close $(T)$ states of prephenate dehydratase (PDT)-implication of allosteric regulation by L-phenylalanine. J Struct Biol 2008; 162: 94-107.

22 Kobe B, Jennings IG, House CM et al. Structural basis of autoregulation of phenylalanine hydroxylase. Nat Struct Biol 1999; 6: 442-448.

23 Lang EJ, Cross PJ, Mittelstädt G, Jameson GB, Parker EJ. Allosteric ACTion: the varied ACT domains regulating enzymes of amino-acid metabolism. Curr Opin Struct Biol 2014; 29: 102-111.

24 Dumas R, Cobessi D, Robin AY, Ferrer J-L, Curien G. The many faces of aspartate kinases. Arch Biochem Biophys 2012; 519: 186-193.

25 Wang S, Tsun ZY, Wolfson RL et al. Metabolism. Lysosomal amino acid transporter SLC38A9 signals arginine sufficiency to mTORC1. Science 2015; 347: 188-194.

26 Rebsamen M, Pochini L, Stasyk T et al. SLC38A9 is a component of the lysosomal amino acid sensing machinery that controls mTORC1. Nature 2015; 519: 477-481.

27 Jung J, Genau HM, Behrends C. Amino acid-dependent mTORC1 regulation by the lysosomal membrane protein SLC38A9. Mol Cell Biol 2015; 35: 2479-2494.

28 Ban $\mathrm{H}$, Shigemitsu K, Yamatsuji T et al. Arginine and leucine regulate p70 S6 kinase and 4E-BP1 in intestinal epithelial cells. Int J Mol Med 2004; 13: 537-543.

29 Fox HL, Pham PT, Kimball SR, Jefferson LS, Lynch CJ. Amino acid effects on translational repressor 4E-BP1 are mediated primarily by L-leucine in isolated adipocytes. $\mathrm{Am}$ J Physiol 1998; 275: C1232-C1238.

30 Kotaka M, Ren J, Lockyer M, Hawkins AR, Stammers DK. Structures of R-and T-state Escherichia coli aspartokinase III. Mechanisms of the allosteric transition and inhibition by lysine. J Biol Chem 2006; 281: 31544-31552.

31 Robin AY, Cobessi D, Curien G et al. A new mode of dimerization of allosteric enzymes with ACT domains revealed by the crystal structure of the aspartate kinase from Cyanobacteria. J Mol Biol 2010; 399: 283-293.

32 Suhre K, Sanejouand YH. ElNémo: a normal mode web server for protein movement analysis and the generation of templates for molecular replacement. Nucleic Acids Res 2004; 32: W610-W614.

33 Floyd JC Jr, Fajans SS, Conn JW, Knopf RF, Rull J. Stimulation of insulin secretion by amino acids. $J$ Clin Invest 1966; 45: 1487-1502.

34 Rhoads JM, Niu X, Odle J, Graves LM. Role of mTOR signaling in intestinal cell migration. Am J Physiol Gastrointest Liver Physiol 2006; 291: G510-G517.

35 Bronte V, Zanovello P. Regulation of immune responses by L-arginine metabolism. Nat Rev Immunol 2005; 5: 641-654. 
36 Zhang Y, Fu L, Qi X et al. Structural insight into the mutual recognition and regulation between suppressor of fused and Gli/Ci. Nat Commun 2013; 4: 2608.

37 Saxton RA, Chantranupong L, Knockenhauer KE, Schwartz TU, Sabatini DM. Mechanism of arginine sensing by CASTOR1 upstream of mTORC1. Nature 2016; 536: 229-233.

38 Xia J, Wang R, Zhang T, Ding J. Structural insight into the arginine-binding specificity of CASTOR 1 in amino acid-dependent mTORC1 signaling. Cell Discov 2016; 2: QJ;16035.

39 Otwinowski Z, Minor W. Processing of X-ray diffraction data collected in oscillation mode. Methods Enzymol 1997; 276: $307-326$.

40 Adams PD, Grosse-Kunstleve RW, Hung LW et al. PHENIX: Building new software for automated crystallographic structure determination. Acta Crystallogr D Biol Crystallogr 2002; 58: 1948-1954.

41 McCoy AJ, Grosse-Kunstleve RW, Adams PD et al. Phaser crystallographic software. J Appl Crystallogr 2007; 40: 658-674.

42 Collaborative Computational Project Number 4. The CCP4 suite: programs for protein crystallography. Acta Crystallogr D Biol Crystallogr 1994; 50: 760-763.

43 Emsley P, Cowtan K. Coot: model-building tools for molecular graphics. Acta Crystallogr D Biol Crystallogr 2004; 60: 2126-2132.

44 Winn MD, Murshudov GN, Papiz MZ. Macromolecular TLS refinement in REFMAC at moderate resolutions. Methods Enzymol 2003; 374: 300-321.
45 Gai Z, Chu W, Deng W et al. Structure of the TBC1D7-TSC1 complex reveals that TBC1D7 stabilizes dimerization of the TSC1 C-terminal coiled coil region. J Mol Cell Biol 2016; 8: 411-425.

46 Zhang Z, Chen L, Gao L et al. Structural basis for the recognition of Asef by adenomatous polyposis coli. Cell Res 2012; 22: 372-386.

47 Schrodinger LLC. The PyMOL Molecular Graphics System, version 13r1, 2010.

48 Landau M, Mayrose I, Rosenberg Y et al. ConSurf 2005: The projection of evolutionary conservation scores of residues on protein structures. Nucleic Acids Res 2005; 33 : W299-W302.

(Supplementary information is linked to the online version of the paper on the Cell Discovery website.)

(c) (i) This work is licensed under a Creative Commons Attribution 4.0 International License. The images or other third party material in this article are included in the article's Creative Commons license, unless indicated otherwise in the credit line; if the material is not included under the Creative Commons license, users will need to obtain permission from the license holder to reproduce the material. To view a copy of this license, visit http://creativecommons.org/licenses/by/4.0/

(C) The Author(s) 2016 